\title{
Synthesis, crystal structure, NMR characterization, Thermal analysis and Spectroscopic Characteristics of $\left[2,3-\left(\mathrm{CH}_{3}\right)_{2} \mathrm{C}_{6} \mathrm{H}_{3} \mathrm{NH}_{3}\right]_{6} \mathrm{P}_{6} \mathrm{O}_{18 .} 2 \mathrm{H}_{2} \mathrm{O}$
}

\author{
R. Fezai*, L. Khedhiri, M. Rzaigui \\ Chemistry Laboratory of Materials LR13ES08, Sciences Faculty of Bizerta, \\ 7021 Jarzouna, Bizerta, Tunisia \\ fezai_ramzi@yahoo.fr \\ Chemistry Laboratory of Materials LR13ES08, Sciences Faculty of Bizerta, \\ 7021 Jarzouna, Bizerta, Tunisia \\ lamia.khedhiri@gmail.com \\ Chemistry Laboratory of Materials LR13ES08, Sciences Faculty of Bizerta, \\ 7021 Jarzouna, Bizerta, Tunisia \\ Mohamed.Rzaigui@fsb.rnu.tn \\ *Corresponding author: fezai_ramzi@yahoo.fr
}

\begin{abstract}
Single crystals of $\left[2,3-\left(\mathrm{CH}_{3}\right)_{2} \mathrm{C}_{6} \mathrm{H}_{3} \mathrm{NH}_{3}\right]_{6} \mathrm{P}_{6} \mathrm{O}_{18} .2 \mathrm{H}_{2} \mathrm{O}$ were synthesized in aqueous solution. This compound crystalizes in the $P \bar{I}$ Triclinic unit cell with the parameters $a=9.97 \AA, b=16.06 \AA, c=18.62$ $\AA$, $\alpha=93.03^{\circ}, \beta=89.75^{\circ}, \gamma=93.85^{\circ}$, and $Z=2$. Its crystal structure, determined by $X$-ray diffraction, consists of inorganic layers parallel to the ac plane. The organic molecules are disposed parallel to the plane bc on either side of each inorganic layer on which are anchored by hydrogen bonds via their ammonium groups $(\mathrm{N}-\mathrm{H} \ldots \mathrm{O})$. Configuration of the asymmetric unit is characterized by $\mathrm{FT}$ IR spectroscopy and solid state NMR study of ${ }^{31} \mathrm{P}$.
\end{abstract}

\section{Keywords:}

Cyclohexaphosphate; X-ray diffraction; Crystal structure; NMR study; Thermal analysis and IR spectroscopy.

\section{Academic Discipline And Sub-Disciplines}

Materials Science

\section{SUBJECT CLASSIFICATION}

Chemistry Subject Classification

\section{TYPE (METHOD/APPROACH)}

Experimental study

\section{Council for Innovative Research}

Peer Review Research Publishing System

Journal: Journal of Advances in Chemistry

Vol. 11, No. 2

editorjaconline@gmail.com

www.cirjac.com 


\section{INTRODUCTION}

The area of framework materials still develops and interest many laboratories not only because of the wide variety of structures but also due to their potential applications in different areas such as catalysis, sorption and separation processes (Mahesh et al., 2002) [1]. Hybrid compounds resulting from the association of organic and inorganic species constitute an important family of these materials.

In the present work, we report synthesis, crystal structure, and characterization by IR spectroscopy, NMR and thermal analysis of the new cyclohexaphosphate of organic cation [2,3$\left.\left(\mathrm{CH}_{3}\right)_{2} \mathrm{C}_{6} \mathrm{H}_{3} \mathrm{NH}_{3}\right]_{6} \mathrm{P}_{6} \mathrm{O}_{18} .2 \mathrm{H}_{2} \mathrm{O}$.

\section{EXPERIMENTAL}

\section{Chemical preparation}

Crystals of the title compound were obtained from acide-base reaction by adding dropwise an ethanolic solution $(10 \mathrm{ml})$ of 2,3 -dimethylaniline $(18 \mathrm{mmol})$ to an aqueous solution (20 $\mathrm{ml})$ of cyclohexaphosphoric acid $(3 \mathrm{mmol})$. The reaction mixture was stirred at room temperature for few minutes. Crystals of the title compound appeared by slow evaporation at room temperature. The cyclohexaphosphoric acid $\mathrm{H}_{6} \mathrm{P}_{6} \mathrm{O}_{18}$, was freshly produced by passing through an ion-exchange resin (Amberlite IR 120) the lithium salt $\mathrm{Li}_{6} \mathrm{P}_{6} \mathrm{O}_{18} .6 \mathrm{H}_{2} \mathrm{O}$, prepared from $\mathrm{LiH}_{2} \mathrm{PO}_{4}$ [2].

\section{$X-$ Ray diffraction}

Single-crystal X-ray diffraction data were collected at $294 \mathrm{~K}$ by using Mach3 Enraf-Nonius diffractometer. Measurement conditions, structure solving and results are summarized in Table 1.

Table 1: Data Collection and Final Results of the Structure Determination

\section{Crystal data}

Formula:

Formula weight:

System:

Space group:

Unit-cell parameters:

Z:

$\mathrm{V}\left(\AA^{3}\right)$

$\mathrm{F}(000)$

Linear absorption factor $\mu\left(\mathrm{mm}^{-1}\right)$

Crystal size $\mathrm{mm}^{3}$

Morphology

II. Intensity measurements

Diffractometer:

Monochromator:

Radiation:

Temperature:

Density (calculated), g.cm ${ }^{-3}$

$\Theta$ range for data collection, ${ }^{\circ} \mathrm{C}$

Absorption Correction: refined from

$\Delta$ F (DIFABS; Walker \& Stuart, 1983) [3]
[2,3- $\left.\left(\mathrm{CH}_{3}\right)_{2} \mathrm{C}_{6} \mathrm{H}_{3} \mathrm{NH}_{3}\right]_{6} \mathrm{P}_{6} \mathrm{O}_{18} .2 \mathrm{H}_{2} \mathrm{O}$

1223.94

Triclinic

PT

$a=9.97 \AA, b=16.06 \AA, c=18.62 \AA$

$\alpha=93.03^{\circ}, \beta=89.75^{\circ}, \gamma=93.85^{\circ}$

2

2971.6

1290

0.138

$(0.70 \times 0.40 \times 0.15)$

Colorless prisms

Enraf-Nonius MACH 3

Graphite

$\mathrm{Ag} \mathrm{K}_{\bar{\alpha}}^{-}(0.5608)$

$293 \mathrm{~K}$

1.368

$2-28$

$\mathrm{T}_{\min }=0.90, \mathrm{~T}_{\max }=0.0 .97$ 
Reflections measured

III. Structure determination

Unique reflections included

Number of parameters refined

Residual Fourier density e $\AA^{-3}$

$R$ indices (all data)

WR

Goodness of fit on F

Computer programs
$28961\left(R_{\text {int }}=0.012\right)$

$8622[(\mathrm{I})>2 \sigma(\mathrm{I})]$

722

$-0.478<\rho<0.578$

0.08

0.23

0.87

Diamond [4], Ortep [5]

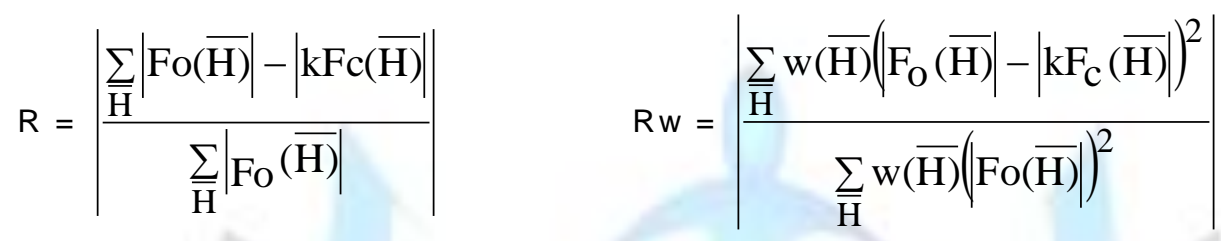

Atomic coordinates and the most significant geometrical characteristics of this structure are reported in Tables 2 and 3 .

Table 2: Atomic Coordinates and $\mathrm{U}_{\mathrm{eq}}$ for $\left[2,3-\left(\mathrm{CH}_{3}\right)_{2} \mathrm{C}_{6} \mathrm{H}_{3} \mathrm{NH}_{3}\right]_{6} \mathrm{P}_{6} \mathrm{O}_{18} .2 \mathrm{H}_{2} \mathrm{O}$

\begin{tabular}{|c|c|c|c|c|}
\hline Atoms & $x(\sigma)$ & $y(\sigma)$ & $z(\sigma)$ & $U_{\text {eq }} / U_{\text {iso }}{ }^{*}$ \\
\hline P1 & $2.27339(8)$ & $0.50956(5)$ & $1.58323(4)$ & $0.02889(18)$ \\
\hline P2 & $2.29341(9)$ & $0.57721(5)$ & $1.43992(5)$ & $0.03009(19)$ \\
\hline P3 & $2.45439(9)$ & $0.46418(5)$ & $1.35602(5)$ & $0.03080(19)$ \\
\hline P4 & $2.23124(8)$ & $0.50351(5)$ & $0.92295(4)$ & $0.02898(18)$ \\
\hline P5 & $2.20959(8)$ & $0.41199(5)$ & $1.05534(4)$ & $0.02962(18)$ \\
\hline P6 & $2.03615(8)$ & $0.51384(5)$ & $1.14485(4)$ & $0.03033(19)$ \\
\hline 01 & $2.2717(2)$ & $0.42051(14)$ & $1.60101(12)$ & $0.0381(5)$ \\
\hline $\mathrm{O} 2$ & $2.1645(2)$ & $0.55901(15)$ & $1.61005(14)$ & $0.0487(7)$ \\
\hline $\mathrm{O} 3$ & $2.2832(3)$ & $0.50836(15)$ & $1.49855(12)$ & $0.0534(7)$ \\
\hline O4 & $2.1763(3)$ & $0.56203(17)$ & $1.39316(15)$ & $0.0572(7)$ \\
\hline O5 & $2.3293(2)$ & $0.66126(14)$ & $1.47323(13)$ & $0.0393(6)$ \\
\hline O6 & $2.4244(2)$ & $0.55040(14)$ & $1.39682(13)$ & $0.0424(6)$ \\
\hline O7 & $2.3496(2)$ & $0.39868(14)$ & $1.37316(13)$ & $0.0399(6)$ \\
\hline O8 & $2.5870(2)$ & $0.44182(15)$ & $1.39564(14)$ & $0.0471(6)$ \\
\hline O9 & $2.4865(3)$ & $0.48029(16)$ & $1.28030(13)$ & $0.0530(7)$ \\
\hline O10 & $2.3371(2)$ & $0.45842(15)$ & $0.88728(14)$ & $0.0488(7)$ \\
\hline 011 & $2.2323(2)$ & $0.59535(14)$ & $0.91682(12)$ & $0.0388(6)$ \\
\hline 012 & $2.2264(3)$ & $0.48995(15)$ & $1.00674(12)$ & $0.0522(7)$ \\
\hline 013 & $2.3225(3)$ & $0.41795(18)$ & $1.10526(15)$ & $0.0590(8)$ \\
\hline 014 & $2.1741(2)$ & $0.33412(14)$ & $1.01235(13)$ & $0.0406(6)$ \\
\hline 015 & $2.0765(2)$ & $0.43300(14)$ & $1.09868(14)$ & $0.0453(6)$ \\
\hline 016 & $2.1410(2)$ & $0.58271(14)$ & $1.13973(12)$ & $0.0374(5)$ \\
\hline
\end{tabular}




\begin{tabular}{|c|c|c|c|c|}
\hline$\overline{017}$ & $1.9124(2)$ & $0.54014(14)$ & $1.10005(13)$ & $0.0421(6)$ \\
\hline 018 & $1.9917(3)$ & $0.48881(16)$ & $1.21660(12)$ & $0.0461(6)$ \\
\hline O19 & $2.1915(6)$ & $0.1637(4)$ & $1.0194(5)$ & $0.217(3)$ \\
\hline O20 & $2.3121(5)$ & $0.8332(3)$ & 1.4892(3) & $0.159(2)$ \\
\hline N1 & $2.4234(3)$ & $0.58179(16)$ & $1.17251(15)$ & $0.0363(6)$ \\
\hline N2 & $2.0929(3)$ & $0.68263(16)$ & $1.02656(15)$ & $0.0380(7)$ \\
\hline N3 & $1.9645(3)$ & $0.61810(15)$ & $1.32591(14)$ & $0.0333(6)$ \\
\hline N4 & $2.3984(3)$ & $0.31696(17)$ & $1.49877(15)$ & $0.0389(7)$ \\
\hline N5 & $2.0716(3)$ & $0.39819(16)$ & $1.33097(15)$ & $0.0361(6)$ \\
\hline N6 & $2.5409(3)$ & $0.36406(16)$ & $1.16619(14)$ & $0.0330(6)$ \\
\hline C1 & $2.4341(3)$ & $0.6720(2)$ & $1.18714(18)$ & $0.0342(7)$ \\
\hline C2 & $2.4631(3)$ & $0.7226(2)$ & $1.13042(19)$ & $0.0364(7)$ \\
\hline C3 & $2.4769(4)$ & $0.8092(2)$ & $1.1459(2)$ & $0.0537(10)$ \\
\hline C4 & $2.4595(5)$ & $0.8392(3)$ & $1.2155(3)$ & $0.0677(13)$ \\
\hline C5 & $2.4275(5)$ & $0.7876(3)$ & $1.2704(2)$ & $0.0692(13)$ \\
\hline C6 & $2.4154(4)$ & $0.7031(2)$ & $1.2563(2)$ & $0.0492(10)$ \\
\hline $\mathrm{C7}$ & $2.4765(4)$ & $0.6870(2)$ & $1.05465(19)$ & $0.0501(10)$ \\
\hline C8 & $2.5114(6)$ & $0.8689(3)$ & $1.0879(3)$ & $0.0835(16)$ \\
\hline C9 & $2.1235(3)$ & $0.7720(2)$ & $1.0405(2)$ & $0.0389(8)$ \\
\hline $\mathrm{C} 10$ & $2.1304(4)$ & $0.8232(2)$ & $0.9830(2)$ & $0.0489(9)$ \\
\hline C11 & $2.1571(4)$ & $0.9100(3)$ & $0.9976(3)$ & $0.0599(12)$ \\
\hline $\mathrm{C} 12$ & $2.1724(5)$ & $0.9384(3)$ & $1.0674(3)$ & $0.0749(15)$ \\
\hline $\mathrm{C} 13$ & $2.1646(5)$ & $0.8872(3)$ & $1.1237(3)$ & $0.0770(15)$ \\
\hline C14 & $2.1397(4)$ & $0.8020(3)$ & $1.1113(2)$ & $0.0567(11)$ \\
\hline C15 & $2.1073(6)$ & $0.7868(3)$ & $0.9083(2)$ & $0.0782(16)$ \\
\hline C16 & $2.1686(6)$ & $0.9681(3)$ & $0.9366(3)$ & $0.112(2)$ \\
\hline C17 & $1.9790(3)$ & $0.7073(2)$ & $1.31540(18)$ & $0.0354(7)$ \\
\hline C18 & $1.8969(4)$ & $0.7428(2)$ & $1.2673(2)$ & $0.0414(8)$ \\
\hline C19 & $1.9142(4)$ & $0.8289(2)$ & $1.2610(2)$ & $0.0547(11)$ \\
\hline C20 & $2.0095(5)$ & $0.8753(3)$ & $1.3035(3)$ & $0.0703(14)$ \\
\hline $\mathrm{C} 21$ & $2.0856(5)$ & $0.8386(3)$ & $1.3512(3)$ & $0.0721(14)$ \\
\hline C22 & $2.0721(4)$ & $0.7539(2)$ & $1.3579(2)$ & $0.0512(10)$ \\
\hline C23 & $1.7931(4)$ & $0.6907(3)$ & $1.2246(2)$ & $0.0626(12)$ \\
\hline C24 & $1.8337(5)$ & $0.8734(3)$ & $1.2086(3)$ & $0.0872(17)$ \\
\hline C25 & $2.3613(4)$ & $0.0871(2)$ & $1.4533(3)$ & $0.0566(11)$ \\
\hline C26 & $2.3863(4)$ & $0.1728(2)$ & $1.4450(2)$ & $0.0473(9)$ \\
\hline C27 & $2.3639(3)$ & $0.2272(2)$ & $1.5042(2)$ & $0.0402(8)$ \\
\hline C28 & $2.3173(5)$ & $0.2004(3)$ & $1.5693(2)$ & $0.0620(12)$ \\
\hline C29 & $2.2937(5)$ & $0.1155(3)$ & $1.5763(3)$ & $0.0812(16)$ \\
\hline C30 & $2.3156(5)$ & $0.0610(3)$ & $1.5197(3)$ & $0.0736(14)$ \\
\hline C31 & $2.4385(5)$ & $0.2066(3)$ & $1.3774(2)$ & $0.0691(13)$ \\
\hline C32 & $2.3797(6)$ & $0.0240(3)$ & $1.3931(3)$ & $0.0929(18)$ \\
\hline
\end{tabular}




\begin{tabular}{|c|c|c|c|c|}
\hline C33 & $2.0520(4)$ & $0.1708(3)$ & $1.3661(3)$ & $0.0606(12)$ \\
\hline C34 & $2.0491(4)$ & $0.2592(2)$ & $1.3793(2)$ & $0.0453(9)$ \\
\hline C35 & $2.0780(3)$ & $0.3072(2)$ & $1.3220(2)$ & $0.0392(8)$ \\
\hline C36 & $2.1205(4)$ & $0.2757(3)$ & $1.2545(2)$ & $0.0569(11)$ \\
\hline C37 & $2.1256(5)$ & $0.1912(3)$ & $1.2443(3)$ & $0.0770(15)$ \\
\hline C38 & $2.0917(5)$ & $0.1407(3)$ & $1.2995(3)$ & $0.0751(15)$ \\
\hline C39 & $2.0142(5)$ & $0.2972(3)$ & $1.4511(2)$ & $0.0670(13)$ \\
\hline $\mathrm{C} 40$ & $2.0120(6)$ & $0.1130(3)$ & $1.4246(3)$ & $0.105(2)$ \\
\hline C41 & $2.6270(5)$ & $0.1572(3)$ & $1.2255(2)$ & $0.0554(11)$ \\
\hline C42 & $2.6320(4)$ & $0.2432(2)$ & $1.21996(18)$ & $0.0378(8)$ \\
\hline $\mathrm{C} 43$ & $2.5380(3)$ & $0.2745(2)$ & $1.17504(18)$ & $0.0351(7)$ \\
\hline C44 & $2.4467(4)$ & $0.2244(3)$ & $1.1351(2)$ & $0.0539(10)$ \\
\hline C45 & $2.4438(5)$ & $0.1398(3)$ & $1.1415(3)$ & $0.0741(15)$ \\
\hline C46 & $2.5324(5)$ & $0.1068(3)$ & $1.1856(3)$ & $0.0704(14)$ \\
\hline C47 & $2.7326(4)$ & $0.3003(3)$ & $1.2600(2)$ & $0.0555(11)$ \\
\hline C48 & $2.7238(6)$ & $0.1161(3)$ & $1.2729(3)$ & $0.0943(19)$ \\
\hline $\mathrm{H} 1 \mathrm{~A}$ & 2.4379 & 0.5703 & 1.1260 & $0.054^{*}$ \\
\hline $\mathrm{H} 1 \mathrm{~B}$ & 2.3414 & 0.5614 & 1.1842 & $0.054^{*}$ \\
\hline $\mathrm{H} 1 \mathrm{C}$ & 2.4842 & 0.5585 & 1.1984 & $0.054^{*}$ \\
\hline $\mathrm{H} 2 \mathrm{~A}$ & 2.0921 & 0.6572 & 1.0679 & $0.057^{*}$ \\
\hline $\mathrm{H} 2 \mathrm{~B}$ & 2.1553 & 0.6620 & 0.9976 & $0.057^{*}$ \\
\hline $\mathrm{H} 2 \mathrm{C}$ & 2.0127 & 0.6741 & 1.0057 & $0.057^{*}$ \\
\hline $\mathrm{H} 3 \mathrm{~A}$ & 2.0240 & 0.6052 & 1.3583 & $0.050^{*}$ \\
\hline H3B & 1.9789 & 0.5900 & 1.2844 & $0.050^{*}$ \\
\hline $\mathrm{H} 3 \mathrm{C}$ & 1.8818 & 0.6045 & 1.3414 & $0.050^{*}$ \\
\hline $\mathrm{H} 4 \mathrm{~A}$ & 2.3567 & -0.0310 & 1.4091 & $0.139^{*}$ \\
\hline $\mathrm{H} 4 \mathrm{~B}$ & 2.4718 & 0.0277 & 1.3776 & $0.139^{*}$ \\
\hline $\mathrm{H} 4 \mathrm{C}$ & 2.3225 & 0.0347 & 1.3537 & $0.139^{*}$ \\
\hline $\mathrm{H} 5 \mathrm{~A}$ & 1.9874 & 0.1452 & 1.4670 & $0.157^{*}$ \\
\hline H5B & 2.0862 & 0.0806 & 1.4354 & $0.157^{*}$ \\
\hline $\mathrm{H} 5 \mathrm{C}$ & 1.9368 & 0.0762 & 1.4089 & $0.157^{*}$ \\
\hline $\mathrm{H} 6 \mathrm{~A}$ & 2.7825 & 0.1583 & 1.2970 & $0.141^{*}$ \\
\hline H6B & 2.7761 & 0.0793 & 1.2437 & $0.141^{*}$ \\
\hline $\mathrm{H} 6 \mathrm{C}$ & 2.6741 & 0.0848 & 1.3078 & $0.141^{*}$ \\
\hline $\mathrm{H} 7 \mathrm{~A}$ & 2.4966 & 0.7316 & 1.0231 & $0.075^{\star}$ \\
\hline H7B & 2.3936 & 0.6571 & 1.0401 & $0.075^{*}$ \\
\hline $\mathrm{H} 7 \mathrm{C}$ & 2.5477 & 0.6496 & 1.0524 & $0.07^{*}$ \\
\hline $\mathrm{H} 8 \mathrm{~A}$ & 2.5214 & 0.8379 & 1.0429 & $0.125^{\star}$ \\
\hline $\mathrm{H} 8 \mathrm{~B}$ & 2.5941 & 0.9006 & 1.0998 & $0.125^{\star}$ \\
\hline $\mathrm{H} 8 \mathrm{C}$ & 2.4407 & 0.9061 & 1.0840 & $0.125^{*}$ \\
\hline $\mathrm{H} 15 \mathrm{~A}$ & 2.1155 & 0.8306 & 0.8750 & $0.117^{*}$ \\
\hline H15B & 2.0188 & 0.7595 & 0.9049 & $0.117^{*}$ \\
\hline
\end{tabular}




\begin{tabular}{|c|c|c|c|c|}
\hline $\mathrm{H} 15 \mathrm{C}$ & 2.1729 & 0.7470 & 0.8969 & $0.117^{*}$ \\
\hline $\mathrm{H} 16 \mathrm{~A}$ & 2.1545 & 0.9364 & 0.8918 & $0.168^{*}$ \\
\hline H16B & 2.2566 & 0.9963 & 0.9369 & $0.168^{*}$ \\
\hline $\mathrm{H} 16 \mathrm{C}$ & 2.1021 & 1.0084 & 0.9423 & $0.168^{*}$ \\
\hline $\mathrm{H} 23 \mathrm{~A}$ & 1.7463 & 0.7256 & 1.1943 & $0.094^{*}$ \\
\hline $\mathrm{H} 23 \mathrm{~B}$ & 1.7303 & 0.6638 & 1.2566 & $0.094^{*}$ \\
\hline $\mathrm{H} 23 \mathrm{C}$ & 1.8358 & 0.6490 & 1.1954 & $0.094^{*}$ \\
\hline $\mathrm{H} 24 \mathrm{~A}$ & 1.7724 & 0.8337 & 1.1832 & $0.131^{*}$ \\
\hline H24B & 1.8933 & 0.9001 & 1.1750 & $0.131^{*}$ \\
\hline $\mathrm{H} 24 \mathrm{C}$ & 1.7840 & 0.9146 & 1.2343 & $0.131^{*}$ \\
\hline $\mathrm{H} 31 \mathrm{~A}$ & 2.4493 & 0.2665 & 1.3829 & $0.104^{*}$ \\
\hline H31B & 2.3762 & 0.1907 & 1.3391 & $0.104^{*}$ \\
\hline $\mathrm{H} 31 \mathrm{C}$ & 2.5238 & 0.1847 & 1.3662 & $0.104^{*}$ \\
\hline H32A & 2.4272 & 0.3266 & 1.4545 & $0.058^{*}$ \\
\hline H32B & 2.4629 & 0.3336 & 1.5302 & $0.058^{*}$ \\
\hline $\mathrm{H} 32 \mathrm{C}$ & 2.3259 & 0.3452 & 1.5083 & $0.058^{*}$ \\
\hline H39A & 1.9979 & 0.2538 & 1.4843 & $0.100^{*}$ \\
\hline H39B & 1.9349 & 0.3273 & 1.4470 & $0.100^{*}$ \\
\hline $\mathrm{H} 39 \mathrm{C}$ & 2.0874 & 0.3348 & 1.4683 & $0.100^{*}$ \\
\hline $\mathrm{H} 40 \mathrm{~A}$ & 2.0921 & 0.4212 & 1.2895 & $0.054^{*}$ \\
\hline $\mathrm{H} 40 \mathrm{~B}$ & 2.1299 & 0.4184 & 1.3646 & $0.054^{*}$ \\
\hline $\mathrm{H} 40 \mathrm{C}$ & 1.9889 & 0.4102 & 1.3442 & $0.054^{*}$ \\
\hline $\mathrm{H} 47 \mathrm{~A}$ & 2.7195 & 0.3570 & 1.2493 & $0.083^{*}$ \\
\hline H47B & 2.8215 & 0.2868 & 1.2458 & $0.083^{*}$ \\
\hline $\mathrm{H} 47 \mathrm{C}$ & 2.7216 & 0.2940 & 1.3107 & $0.083^{*}$ \\
\hline $\mathrm{H} 48 \mathrm{~A}$ & 2.6040 & 0.3900 & 1.1946 & $0.050^{*}$ \\
\hline $\mathrm{H} 48 \mathrm{~B}$ & 2.4612 & 0.3826 & 1.1780 & $0.050^{*}$ \\
\hline $\mathrm{H} 48 \mathrm{C}$ & 2.5593 & 0.3744 & 1.1206 & $0.050^{*}$ \\
\hline $\mathrm{H} 4$ & 2.4697 & 0.8966 & 1.2258 & $0.081^{*}$ \\
\hline H5 & 2.4142 & 0.8100 & 1.3168 & $0.083^{*}$ \\
\hline $\mathrm{H} 6$ & 2.3948 & 0.6673 & 1.2929 & $0.059^{*}$ \\
\hline $\mathrm{H} 12$ & 2.1891 & 0.9956 & 1.0772 & $0.090^{*}$ \\
\hline $\mathrm{H} 13$ & 2.1761 & 0.9097 & 1.1704 & $0.092^{*}$ \\
\hline $\mathrm{H} 14$ & 2.1340 & 0.7661 & 1.1490 & $0.068^{*}$ \\
\hline $\mathrm{H} 2 \mathrm{O}$ & 2.0212 & 0.9327 & 1.2991 & $0.084^{*}$ \\
\hline $\mathrm{H} 21$ & 2.1475 & 0.8711 & 1.3796 & $0.086^{*}$ \\
\hline H22 & 2.1246 & 0.7285 & 1.3904 & $0.061^{*}$ \\
\hline $\mathrm{H} 44$ & 2.3879 & 0.2473 & 1.1043 & $0.065^{\star}$ \\
\hline $\mathrm{H} 45$ & 2.3811 & 0.1047 & 1.1156 & $0.089^{\star}$ \\
\hline $\mathrm{H} 46$ & 2.5297 & 0.0492 & 1.1892 & $0.085^{\star}$ \\
\hline $\mathrm{H} 28$ & 2.3021 & 0.2383 & 1.6074 & $0.074^{*}$ \\
\hline $\mathrm{H} 29$ & 2.2628 & 0.0956 & 1.6198 & $0.097^{*}$ \\
\hline
\end{tabular}




\begin{tabular}{lllll}
\hline $\mathrm{H} 30$ & 2.2994 & 0.0040 & 1.5256 & $0.088^{\star}$ \\
$\mathrm{H} 36$ & 2.1444 & 0.3112 & 1.2180 & $0.068^{\star}$ \\
$\mathrm{H} 37$ & 2.1519 & 0.1680 & 1.2001 & $0.092^{\star}$ \\
$\mathrm{H} 38$ & 2.0958 & 0.0833 & 1.2915 & $0.090^{\star}$
\end{tabular}

$U_{e q}=4 / 3 \sum_{i} \sum_{j} \beta_{i j} a_{i} a_{j}$

Table 3: Principal Intermolecular Distances $(\AA ̊)$ and bond angles $\left(^{\circ}\right)$

in $\left[2,3-\left(\mathrm{CH}_{3}\right)_{2} \mathrm{C}_{6} \mathrm{H}_{3} \mathrm{NH}_{3}\right]_{6} \mathrm{P}_{6} \mathrm{O}_{18} .2 \mathrm{H}_{2} \mathrm{O}$

\section{$\mathrm{PO}_{4}$ Tetrahedron}

\begin{tabular}{|c|c|c|c|c|}
\hline$P(1)$ & $\mathrm{O}(1)$ & $\mathrm{O}(2)$ & $\mathrm{O}(3)$ & $\mathrm{O}(8)$ \\
\hline $\mathrm{O}(1)$ & $1.483(2)$ & $2.530(4)$ & 2.429(3) & $2.535(4)$ \\
\hline $\mathrm{O}(2)$ & $118.73(14)$ & $1.460(2)$ & $2.511(8)$ & $2.478(4)$ \\
\hline $\mathrm{O}(3)$ & $105.06(13)$ & $111.39(16)$ & $1.557(2)$ & $2.428(2)$ \\
\hline $\mathrm{O}(8)$ & $111.21(13)$ & $108.63(15)$ & $100.22(15)$ & $1.587(2)$ \\
\hline$P(2)$ & $\mathrm{O}(3)$ & $\mathrm{O}(4)$ & $\mathrm{O}(5)$ & $\mathrm{O}(6)$ \\
\hline $\mathrm{O}(3)$ & $1.594(2)$ & $2.462(3)$ & $2.536(4)$ & $2.449(3)$ \\
\hline $\mathrm{O}(4)$ & $107.32(16)$ & $1.460(2)$ & $2.558(3)$ & $2.496(3)$ \\
\hline $\mathrm{O}(5)$ & $111.37(13)$ & $120.98(15)$ & $1.477(2)$ & $2.458(2)$ \\
\hline $\mathrm{O}(6)$ & $100.22(15)$ & $109.25(15)$ & $105.71(13)$ & $1.601(2)$ \\
\hline$P(3)$ & $\mathrm{O}(6)$ & $\mathrm{O}(7)$ & $\mathrm{O}(8)$ & $\mathrm{O}(9)$ \\
\hline $\mathrm{O}(6)$ & $1.590(2)$ & $2.514(2)$ & $2,460(3)$ & $2.488(3)$ \\
\hline $\mathrm{O}(7)$ & $110.35(13)$ & 1. $477(2)$ & $2.554(4)$ & $2.560(3)$ \\
\hline $\mathrm{O}(8)$ & $101.45(14)$ & $106.15(14)$ & $1.591(2)$ & $2.499(2)$ \\
\hline $\mathrm{O}(9)$ & $108.37(14)$ & $119.92(15)$ & $109.01(16)$ & $1.477(2)$ \\
\hline$P(4)$ & $O(10)$ & $\mathrm{O}(11)$ & $\mathrm{O}(12)$ & $\mathrm{O}(17)$ \\
\hline $\mathrm{O}(10)$ & $1.461(6)$ & $2.534(2)$ & $2.525(2)$ & $2.499(2)$ \\
\hline $\mathrm{O}(11)$ & $118.68(14)$ & $1.485(2)$ & $2.444(2)$ & $2.533(3)$ \\
\hline $\mathrm{O}(12)$ & $111.93(16)$ & $105.25(13)$ & $1.485(2)$ & $2.432(3)$ \\
\hline $\mathrm{O}(17)$ & $109.32(14)$ & $110.47(13)$ & $99.45(14)$ & $1.596(2)$ \\
\hline$P(5)$ & $\mathrm{O}(12)$ & $\mathrm{O}(13)$ & $\mathrm{O}(14)$ & $\mathrm{O}(15)$ \\
\hline $\mathrm{O}(12)$ & $1.582(2)$ & $2.456(2)$ & $2.530(3)$ & $2.441(3)$ \\
\hline $\mathrm{O}(13)$ & $107.57(16)$ & $1.461(3)$ & $2.552(2)$ & $2.487(2)$ \\
\hline $\mathrm{O}(14)$ & $111.54(13)$ & $120.95(15)$ & $1.471(2)$ & $2.443(2)$ \\
\hline $\mathrm{O}(15)$ & $100.34(15)$ & $109.00(16)$ & $105.45(14)$ & $1.592(2)$ \\
\hline
\end{tabular}




\begin{tabular}{lllll}
\hline $\mathrm{P}(6)$ & $\mathrm{O}(15)$ & $\mathrm{O}(16)$ & $\mathrm{O}(17)$ & $\mathrm{O}(18)$ \\
$\mathrm{O}(15)$ & $\mathbf{1 . 5 9 6 ( 2 )}$ & $2.527(2)$ & $2.456(2)$ & $2.495(2)$ \\
$\mathrm{O}(16)$ & $110.71(13)$ & $\mathbf{1 . 4 7 8 ( 2 )}$ & $2.442(4)$ & $2.540(3)$ \\
$\mathrm{O}(17)$ & $101.12(14)$ & $105.67(13)$ & $\mathbf{1 . 5 9 1 ( 2 )}$ & $2.515(3)$ \\
$\mathrm{O}(18)$ & $108.62(14)$ & $118.86(15)$ & $110.39(14)$ & $\mathbf{1 . 4 7 1 ( 2 )}$
\end{tabular}

$\begin{array}{llll}\text { P1-P2 } & 2.885(3) & \text { P1-O8-P3 } & 135 \\ \text { P2-P3 } & 2.924(3) & \text { P2-O3-P1 } & 135 \\ \text { P1-P3 } & 2.926(3) & \text { P2-O6-P3 } & 130 \\ \text { P4-P5 } & 2.9379(19) & \text { P6-O17-P4 } & 135 \\ \text { P5-P6 } & 2.9157(18) & \text { P5-O12-P4 } & 135 \\ \text { P4-P6 } & 2.900(3) & \text { P6-O15-P5 } & 132 \\ & & & \\ \text { P1-P2-P3 } & 120.4(8) & \text { P6-P5-P4 } & 109.1(8) \\ \text { P2-P3-P1 } & 99.04(8) & \text { P4-P6-P5 } & 123.1(8) \\ \text { P3-P1-P2 } & 110.3(8) & \text { P5-P4-P6 } & 98.9(8)\end{array}$

\section{Organic groups}

$N(1)-C(1)$

$C(1)-C(2)$

$C(1)-C(6)$

$C(2)-C(3)$

$C(2)-C(7)$

$\mathrm{C}(3)-\mathrm{C}(4)$

$C(3)-C(8)$

$C(4)-C(5)$

$C(5)-C(6)$
1.457(4)

$1.385(5)$

$1.373(5)$

$1.402(5)$

$1.503(5)$

$1.373(6)$

$1.506(6)$

$1.374(6)$

$1.374(6)$

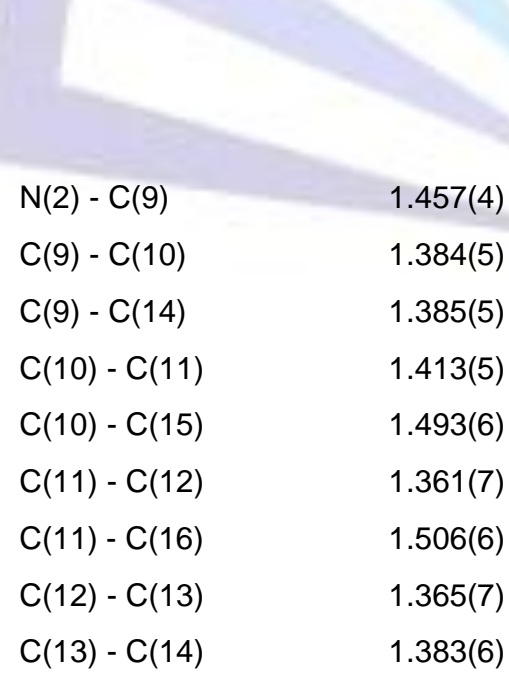

$\mathrm{C}(2)-\mathrm{C}(1)-\mathrm{N}(1)$

$\mathrm{C}(6)-\mathrm{C}(1)-\mathrm{N}(1)$

$C(6)-C(1)-C(2)$

$C(1)-C(2)-C(3)$

$\mathrm{C}(1)-\mathrm{C}(2)-\mathrm{C}(7)$

$\mathrm{C}(3)-\mathrm{C}(2)-\mathrm{C}(7)$

$C(2)-C(3)-C(4)$

$C(2)-C(3)-C(8)$

$C(4)-C(3)-C(8)$

$C(3)-C(4)-C(5)$

$C(4)-C(5)-C(6)$

$C(5)-C(6)-C(1)$

$\mathrm{C}(10)-\mathrm{C}(9)-\mathrm{N}(2)$

$\mathrm{C}(14)-\mathrm{C}(9)-\mathrm{N}(2)$

$C(14)-C(9)-C(10)$

$C(9)-C(10)-C(11)$

$C(9)-C(10)-C(15)$

$C(11)-C(10)-C(15)$

$C(10)-C(11)-C(12)$

$C(10)-C(11)-C(16)$

$C(12)-C(11)-C(16)$

$C(11)-C(12)-C(13)$

$C(12)-C(13)-C(14)$

$C(13)-C(14)-C(9)$
135.42(16)

$135.49(16)$

$130.28(15)$

$135.05(15)$

$135.75(16)$

$132.37(15)$

$98.9(8)$

118.3(3)

$118.8(3)$

122.9(3)

$117.5(3)$

$121.7(3)$

$120.8(3)$

$118.9(4)$

$121.1(4)$

$120.0(4)$

$122.4(4)$

$119.4(4)$

$119.0(4)$

118.7(3)

118.1(3)

123.1(4)

118.0(4)

120.1(4)

$121.8(4)$

$118.2(4)$

$120.0(5)$

$121.9(5)$

123.2(4)

$120.1(5)$

117.4(4) 


\begin{tabular}{|c|c|c|c|}
\hline$N(3)-C(17)$ & $1.454(4)$ & $\mathrm{C}(18)-\mathrm{C}(17)-\mathrm{N}(3)$ & $120.4(3)$ \\
\hline$C(17)-C(18)$ & $1.386(5)$ & $C(22)-C(17)-N(3)$ & $116.8(3)$ \\
\hline$C(17)-C(22)$ & $1.378(5)$ & $C(18)-C(17)-C(22)$ & $122.7(3)$ \\
\hline$C(18)-C(19)$ & $1.394(5)$ & $C(17)-C(18)-C(19)$ & $117.5(4)$ \\
\hline$C(18)-C(23)$ & $1.492(5)$ & $C(17)-C(18)-C(23)$ & $121.1(3)$ \\
\hline$C(19)-C(20)$ & $1.391(6)$ & $C(19)-C(18)-C(23)$ & $121.4(4)$ \\
\hline$C(19)-C(24)$ & $1.504(6)$ & $C(18)-C(19)-C(20)$ & $119.4(4)$ \\
\hline$C(20)-C(21)$ & $1.354(6)$ & $C(18)-C(19)-C(24)$ & $121.9(4)$ \\
\hline \multirow[t]{4}{*}{$C(21)-C(22)$} & $1.369(6)$ & $C(20)-C(19)-C(24)$ & $118.8(4)$ \\
\hline & & $C(19)-C(20)-C(21)$ & $121.4(4)$ \\
\hline & & $C(20)-C(21)-C(22)$ & $120.5(4)$ \\
\hline & & $C(21)-C(22)-C(17)$ & $118.5(4)$ \\
\hline$N(4)-C(25)$ & $1.468(4)$ & $C(26)-C(25)-N(4)$ & $119.9(3)$ \\
\hline$C(25)-C(26)$ & $1.396(5)$ & $C(30)-C(25)-N(4)$ & $121.0(4)$ \\
\hline$C(25)-C(30)$ & $1.376(5)$ & $C(30)-C(25)-C(26)$ & $119.1(4)$ \\
\hline$C(26)-C(27)$ & $1.399(5)$ & $C(25)-C(26)-C(27)$ & $120.2(5)$ \\
\hline$C(26)-C(31)$ & $1.477(5)$ & $C(25)-C(26)-C(31)$ & $118.4(5)$ \\
\hline$C(27)-C(28)$ & $1.391(6)$ & $C(27)-C(26)-C(31)$ & $122.4(5)$ \\
\hline$C(27)-C(32)$ & $1.491(6)$ & $C(26)-C(27)-C(28)$ & $119.6(6)$ \\
\hline$C(28)-C(29)$ & $1.361(7)$ & $C(26)-C(27)-C(32)$ & $118.0(6)$ \\
\hline \multirow[t]{4}{*}{$C(29)-C(30)$} & $1.380(6)$ & $C(28)-C(27)-C(32)$ & $119.7(5)$ \\
\hline & & $C(27)-C(28)-C(29)$ & $118.4(5)$ \\
\hline & & $C(28)-C(29)-C(30)$ & $118.4(5)$ \\
\hline & & $C(29)-C(30)-C(25)$ & $118.4(5)$ \\
\hline$N(5)-C(33)$ & $1.468(4)$ & $C(34)-C(33)-N(5)$ & 119.3(3) \\
\hline$C(33)-C(34)$ & $1.367(5)$ & $C(38)-C(33)-N(5)$ & $116.4(3)$ \\
\hline$C(33)-C(38)$ & $1.405(5)$ & $C(38)-C(33)-C(34)$ & $124.2(4)$ \\
\hline$C(34)-C(35)$ & $1.430(5)$ & $C(33)-C(34)-C(35)$ & $116.4(4)$ \\
\hline$C(34)-C(39)$ & $1.490(5)$ & $C(33)-C(34)-C(39)$ & $121.6(4)$ \\
\hline$C(35)-C(36)$ & $1.374(6)$ & $C(35)-C(34)-C(39)$ & $122.0(4)$ \\
\hline$C(35)-C(40)$ & $1.505(6)$ & $C(34)-C(35)-C(36)$ & $118.6(4)$ \\
\hline$C(36)-C(37)$ & $1.370(7)$ & $C(34)-C(35)-C(40)$ & $119.9(5)$ \\
\hline \multirow[t]{4}{*}{$C(37)-C(38)$} & $1.364(6)$ & $C(36)-C(35)-C(40)$ & $121.5(5)$ \\
\hline & & $C(35)-C(36)-C(37)$ & $123.2(4)$ \\
\hline & & $C(36)-C(37)-C(38)$ & $119.7(5)$ \\
\hline & & $C(37)-C(38)-C(33)$ & $117.7(4)$ \\
\hline$N(6)-C(41)$ & $1.455(4)$ & $C(42)-C(41)-N(6)$ & $119.2(3)$ \\
\hline$C(41)-C(42)$ & $1.395(5)$ & $\mathrm{C}(46)-\mathrm{C}(41)-\mathrm{N}(6)$ & $117.6(3)$ \\
\hline
\end{tabular}




$\begin{array}{llll}\mathrm{C}(41)-\mathrm{C}(46) & 1.368(5) & \mathrm{C}(46)-\mathrm{C}(41)-\mathrm{C}(42) & 123.0(3) \\ \mathrm{C}(42)-\mathrm{C}(43) & 1.387(5) & \mathrm{C}(41)-\mathrm{C}(42)-\mathrm{C}(43) & 117.1(3) \\ \mathrm{C}(42)-\mathrm{C}(47) & 1.488(5) & \mathrm{C}(41)-\mathrm{C}(42)-\mathrm{C}(47) & 120.9(3) \\ \mathrm{C}(43)-\mathrm{C}(44) & 1.392(6) & \mathrm{C}(43)-\mathrm{C}(42)-\mathrm{C}(47) & 122.0(4) \\ \mathrm{C}(43)-\mathrm{C}(48) & 1.517(6) & \mathrm{C}(42)-\mathrm{C}(43)-\mathrm{C}(44) & 119.4(4) \\ \mathrm{C}(44)-\mathrm{C}(45) & 1.360(7) & \mathrm{C}(42)-\mathrm{C}(43)-\mathrm{C}(48) & 121.8(4) \\ \mathrm{C}(45)-\mathrm{C}(46) & 1.368(6) & \mathrm{C}(44)-\mathrm{C}(43)-\mathrm{C}(48) & 118.8(4) \\ & & \mathrm{C}(43)-\mathrm{C}(44)-\mathrm{C}(45) & 121.7(4) \\ & & \mathrm{C}(44)-\mathrm{C}(45)-\mathrm{C}(46) & 120.0(4) \\ & & \mathrm{C}(45)-\mathrm{C}(46)-\mathrm{C}(41) & 118.7(4)\end{array}$

\section{RESULTS AND DISCUSSION}

\section{Structure description}

Asymmetric unity which constitutes the basic entity of this structure has the geometrical configuration depicted in the Ortep representation (Fig. 1).

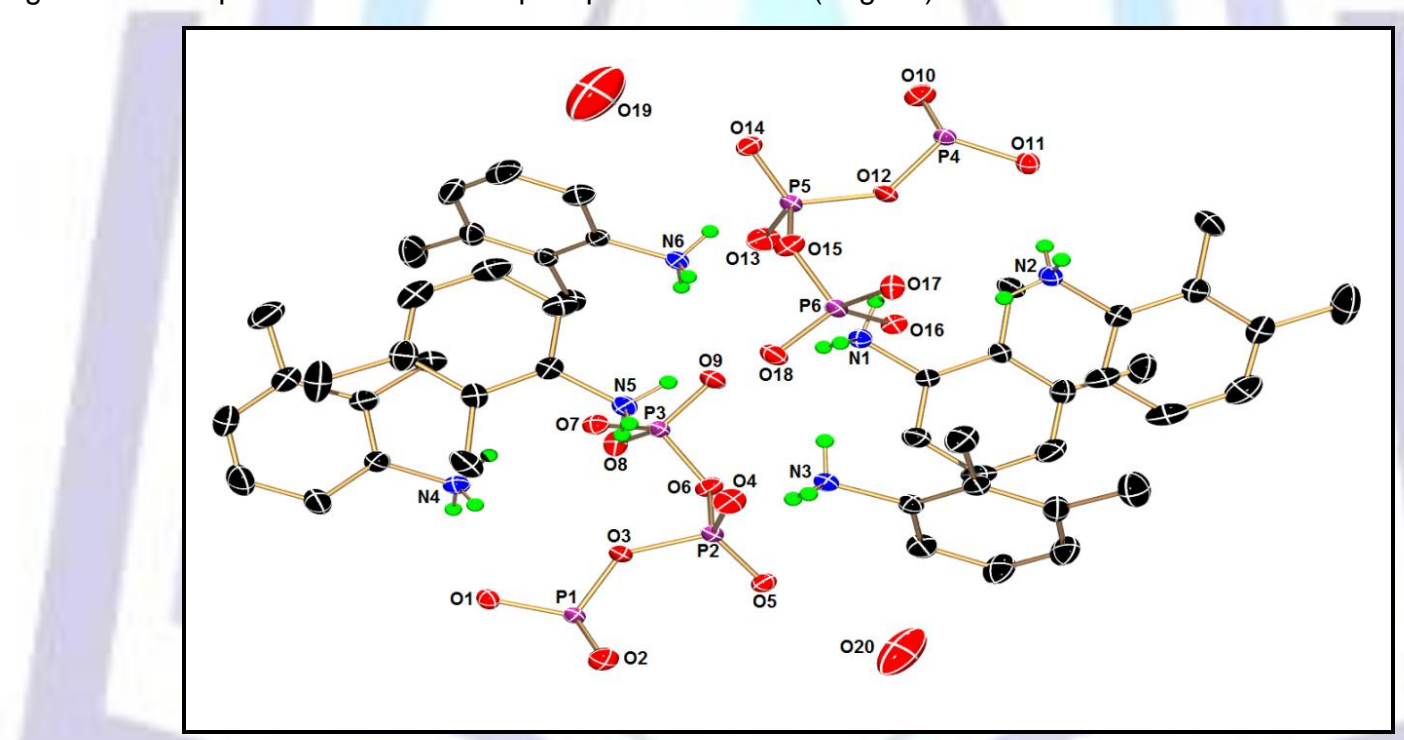

Figure 1: ORTEP Plot of the asymmetric unit of the title structure.

Thermal ellipsoids at $30 \%$ of probability.

This unit generates by symmetry the structure of the title compound (Fig. 2). This projection shows that the atomic arrangement of this compound can be considered as a typical layers organization, were the hexaphosphoric rings, interconnected by $\mathrm{H}$-bonds, adopt a chair conformation and form an inorganic layer parallel to the ac plane. Dimethylanilinium Cations [2,3$\left.\left(\mathrm{CH}_{3}\right)_{2} \mathrm{C}_{6} \mathrm{H}_{3} \mathrm{NH}_{3}\right]^{+}$, obtained by protonation of the amine and presented in the mesh in six crystallographically independent groups, are located between the inorganic layers which are spaced $16.06 \AA(=\mathrm{b})$. These organic entities establish $\mathrm{H}$-bonds of type $\mathrm{N}-\mathrm{H} \ldots \mathrm{O}$ with $[\mathrm{P} 6 \mathrm{O} 18]^{6-}$ rings (listed in table 4) and develop electrostatic and Van Der Walls interactions for ensuring the cohesion and the stability of the crystal framework (Fig. 3).

It must be noted that both water molecules $O\left(W_{1}\right)$ and $O(W 2)$ are not involved in the inorganic layers which may explain their high thermal factors. Such thermal factor values were observed too in others structures (M. Bagieu-Beucher et al., 1991) [6]. 


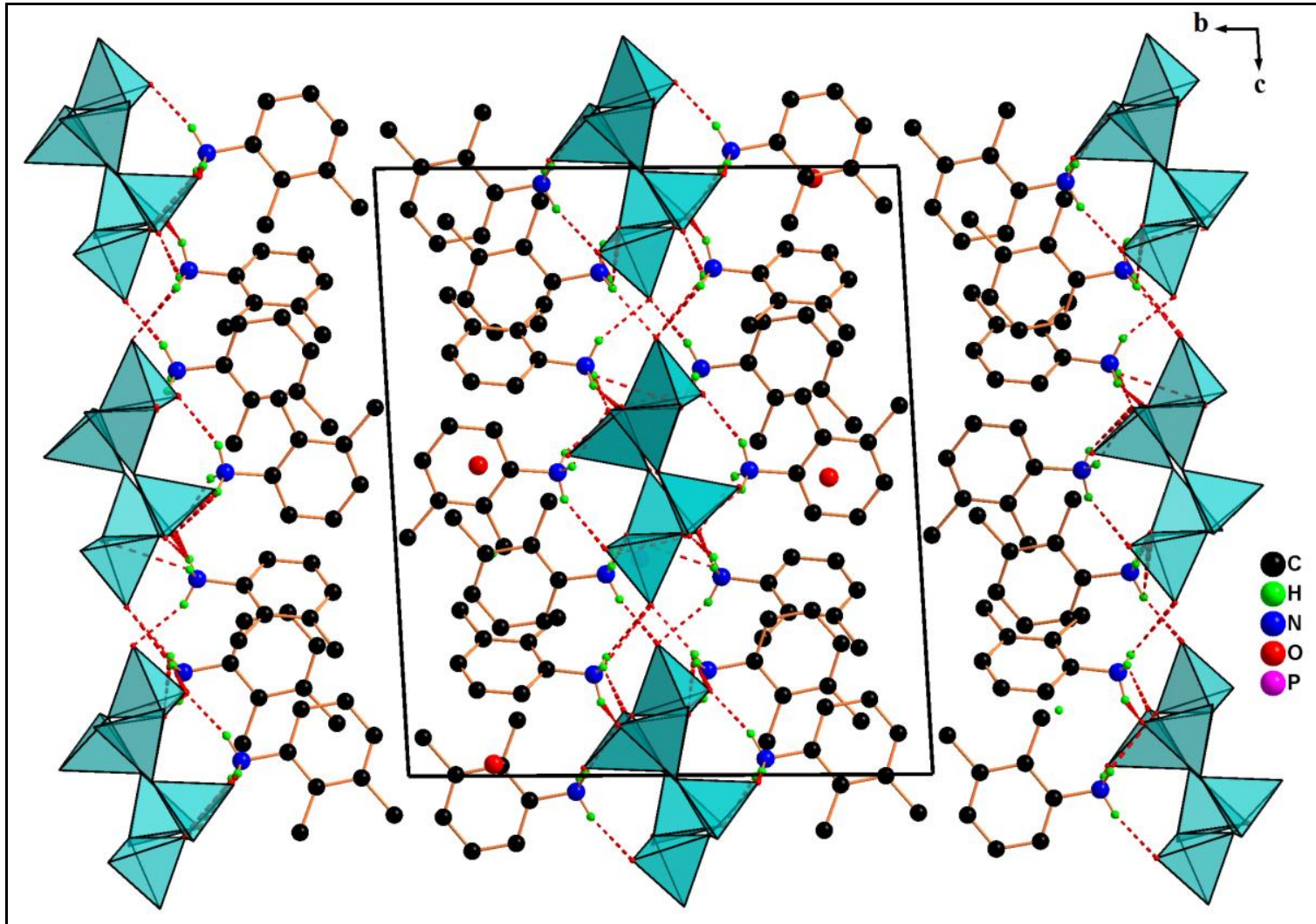

Figure 2: Projection of the crystal structure of $\left[2,3-\left(\mathrm{CH}_{3}\right)_{2} \mathrm{C}_{6} \mathrm{H}_{3} \mathrm{NH}_{3}\right]_{6} \mathrm{P}_{6} \mathrm{O}_{18} .2 \mathrm{H}_{2} \mathrm{O}$, along the a axis, The phosphoric anions are given in tetrahedral representation .

Hydrogen bonds are shown as dashed lines.

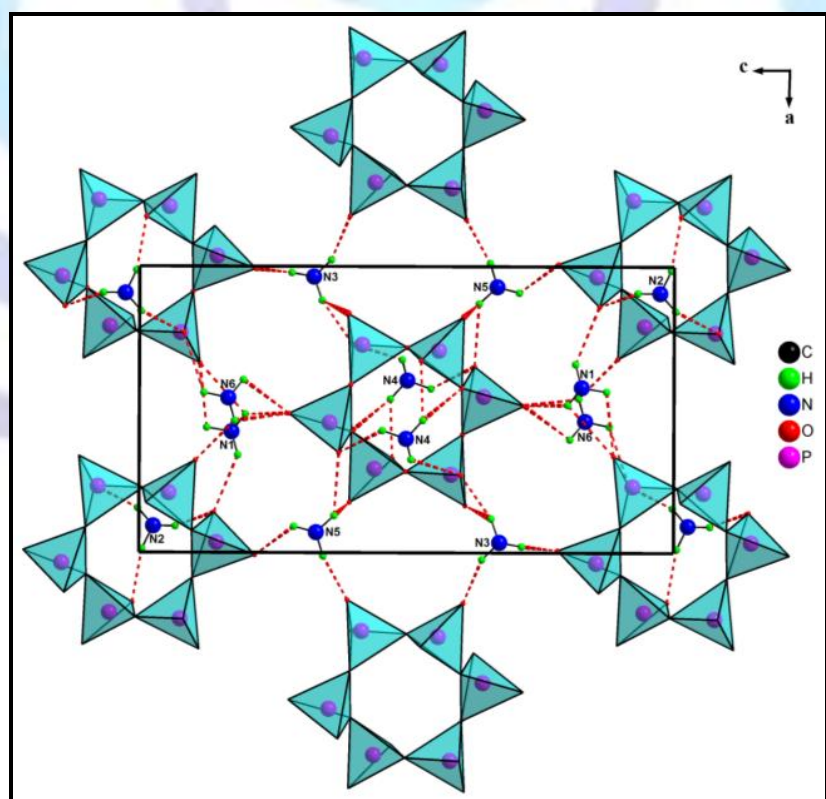

Figure 3: Projection along the $b$ axis of the atomic arrangement of 2,3- $\left.\left(\mathrm{CH}_{3}\right)_{2} \mathrm{C}_{6} \mathrm{H}_{3} \mathrm{NH}_{3}\right]_{6} \mathrm{P}_{6} \mathrm{O}_{18} .2 \mathrm{H}_{2} \mathrm{O}$. Phosphoric anions are given in a tetrahedral representation. The organic cations have been rendered ammonium group for clarity. Hydrogen bonds are plotted by dashed lines. 
Table 4: Hydrogen-bonds geometry $\left(\AA,^{\circ}\right)$ in $\left[2,3-\left(\mathrm{CH}_{3}\right)_{2} \mathrm{C}_{6} \mathrm{H}_{3} \mathrm{NH}_{3}\right]_{6} \mathrm{P}_{6} \mathrm{O}_{18.2} \mathrm{H}_{2} \mathrm{O}$

\begin{tabular}{|c|c|c|c|c|}
\hline Atoms & D - H & H...A & D...A & D - H...A \\
\hline$N(1)-H(1 A) \ldots O(10)^{i i i}$ & 0.89 & 2.33 & $2.733(4)$ & 108 \\
\hline $\mathrm{N}(1)-\mathrm{H}(1 \mathrm{~B}) \ldots \mathrm{O}(16)$ & 0.89 & 2.22 & $2.885(4)$ & 131 \\
\hline$N(1)-H(1 C) \ldots O(9)$ & 0.89 & 2.03 & $2.753(4)$ & 138 \\
\hline$N(1)-H(1 C) \ldots O(10)^{i i i}$ & 0.89 & 2.41 & $2.733(4)$ & 102 \\
\hline $\mathrm{N}(2)-\mathrm{H}(2 \mathrm{~A}) \ldots \mathrm{O}(16)$ & 0.89 & 1.93 & $2.778(4)$ & 160 \\
\hline $\mathrm{N}(2)-\mathrm{H}(2 \mathrm{~A}) \ldots \mathrm{O}(17)^{\mathrm{i}}$ & 0.89 & 2.60 & $3.179(4)$ & 124 \\
\hline $\mathrm{N}(2)-\mathrm{H}(2 \mathrm{~B}) \ldots \mathrm{O}(11)$ & 0.89 & 1.98 & $2.830(4)$ & 158 \\
\hline $\mathrm{N}(2)-\mathrm{H}(2 \mathrm{C}) \ldots \mathrm{O}(14)^{\mathrm{i}}$ & 0.89 & 1.89 & $2.754(4)$ & 164 \\
\hline $\mathrm{N}(3)-\mathrm{H}(3 \mathrm{~A}) \ldots \mathrm{O}(4)^{\mathrm{ii}}$ & 0.89 & 1.85 & $2.690(4)$ & 158 \\
\hline $\mathrm{N}(3)-\mathrm{H}(3 \mathrm{~B}) \ldots \mathrm{O}(18)^{\mathrm{ii}}$ & 0.89 & 2.02 & $2.855(3)$ & 157 \\
\hline $\mathrm{N}(3)-\mathrm{H}(3 \mathrm{C}) \ldots \mathrm{O}(2)$ & 0.89 & 2.83 & $3.321(3)$ & 115 \\
\hline $\mathrm{N}(3)-\mathrm{H}(3 \mathrm{C}) \ldots \mathrm{O}(1)$ & 0.89 & 1.90 & $2.766(4)$ & 165 \\
\hline $\mathrm{N}(4)-\mathrm{H}(32 \mathrm{~A}) \ldots \mathrm{O}(7)$ & 0.89 & 2.13 & $2.802(4)$ & 131 \\
\hline $\mathrm{N}(4)-\mathrm{H}(32 \mathrm{~B}) \ldots \mathrm{O}(5)^{\mathrm{iv}}$ & 0.89 & 2.07 & $2.763(4)$ & 134 \\
\hline $\mathrm{N}(4)-\mathrm{H}(32 \mathrm{~B}) \ldots \mathrm{O}(6)^{\mathrm{iv}}$ & 0.89 & 2.46 & $3.245(4)$ & 148 \\
\hline $\mathrm{N}(4)-\mathrm{H}(32 \mathrm{C}) \ldots \mathrm{O}(1)$ & 0.89 & 2.14 & $2.813(4)$ & 131 \\
\hline$N(5)-H(40 A) \ldots O(18)$ & 0.89 & 2.08 & $2.791(4)$ & 136 \\
\hline $\mathrm{N}(5)-\mathrm{H}(40 \mathrm{~B}) \ldots \mathrm{O}(4)$ & 0.89 & 2.35 & $2.945(4)$ & 124 \\
\hline $\mathrm{N}(5)-\mathrm{H}(40 \mathrm{~B}) \ldots \mathrm{O}(7)$ & 0.89 & 2.24 & $2.884(4)$ & 129 \\
\hline $\mathrm{N}(5)-\mathrm{H}(40 \mathrm{C}) \ldots \mathrm{O}(2)^{\mathrm{ii}}$ & 0.89 & 1.83 & $2.709(4)$ & 168 \\
\hline$N(6)-H(48 A) \ldots O(9)$ & 0.89 & 2.45 & $2.829(4)$ & 106 \\
\hline $\mathrm{N}(6)-\mathrm{H}(48 \mathrm{~B}) \ldots \mathrm{O}(9)$ & 0.89 & 2.41 & $2.829(4)$ & 109 \\
\hline $\mathrm{N}(6)-\mathrm{H}(48 \mathrm{~B}) \ldots \mathrm{O}(13)$ & 0.89 & 2.07 & $2.675(4)$ & 125 \\
\hline $\mathrm{N}(6)-\mathrm{H}(48 \mathrm{C}) \ldots \mathrm{O}(11)^{\mathrm{iii}}$ & 0.89 & 2.22 & $2.794(4)$ & 122 \\
\hline
\end{tabular}

Symmetry codes : i : -x,1-y,-z; ii : -x,1-y,1-z; iii : 1-x,1-y,-z ; iv : 1-x,1-y,1-z

\section{NMR Results}

Crystallographic study of the two phosphoric rings showed a slight difference in their geometry (Fig.4). The gap is close to $0.88^{\circ}$. To evaluate these results we have studied the solid state NMR of ${ }^{31} \mathrm{P}$ of the title compound. The CP/MAS-NMR experiments were performed at room temperature on a Bruker MSL 300 solid state high-resolution spectrometer operating at 121.495 $\mathrm{MHz}$ for ${ }^{31} \mathrm{P}$. Chemical shift values are given with respect to $85 \% \mathrm{H}_{3} \mathrm{PO}_{4}$ (negative shifts are to high field). The ${ }^{31} \mathrm{P}$ MAS-NMR spectrum of crystalline cyclohexaphosphate [2,3$\left.\left(\mathrm{CH}_{3}\right)_{2} \mathrm{C}_{6} \mathrm{H}_{3} \mathrm{NH}_{3}\right]_{6} \mathrm{P}_{6} \mathrm{O}_{18} .2 \mathrm{H}_{2} \mathrm{O}$ is reported in Fig.5. It exhibits four resonance peaks at: -10.67 , $19.8,-23.43$ and $-26.57 \mathrm{ppm}$. The existence of only 4 peaks shows that some crystallographically independent phosphorous of the two rings have very similar chemical environments. Analysis of geometrical characteristics of the six tetrahedra $\mathrm{PO}_{4}$ shows effectively a chemical environment similarity of P2 with P5 and P3 with P6. This similarity gives two NMR peaks for these phosphorous with two others peaks for P1 and P4. The presence of this number of peaks, indicate that the title compound contains more than three crystallography independent phosphoric sites, and therefore two independent $\mathrm{P}_{6} \mathrm{O}_{18}$ rings, which is in agreement with $x$-ray results. These chemical shift values agree with those of others cyclohexaphosphates [7]. 


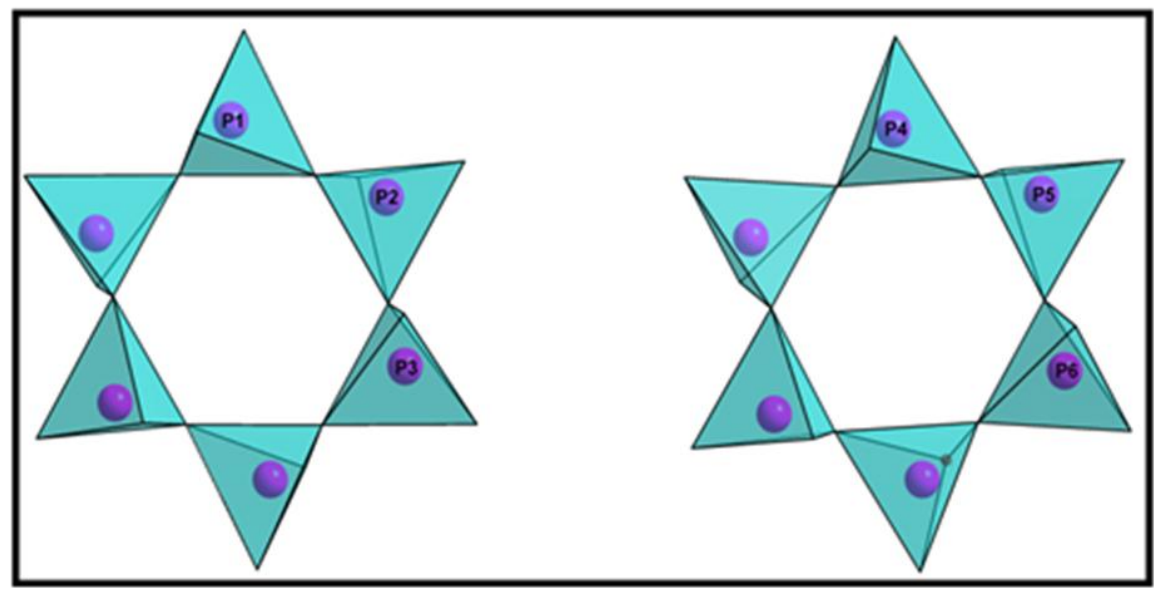

Figure 4: Ggeometry representation of the two $\mathrm{P}_{6} \mathrm{O}_{18}$ rings in the structure of 2,3- $\left.\left(\mathrm{CH}_{3}\right)_{2} \mathrm{C}_{6} \mathrm{H}_{3} \mathrm{NH}_{3}\right]_{6} \mathrm{P}_{6} \mathrm{O}_{18.2} \mathrm{H}_{2} \mathrm{O}$.

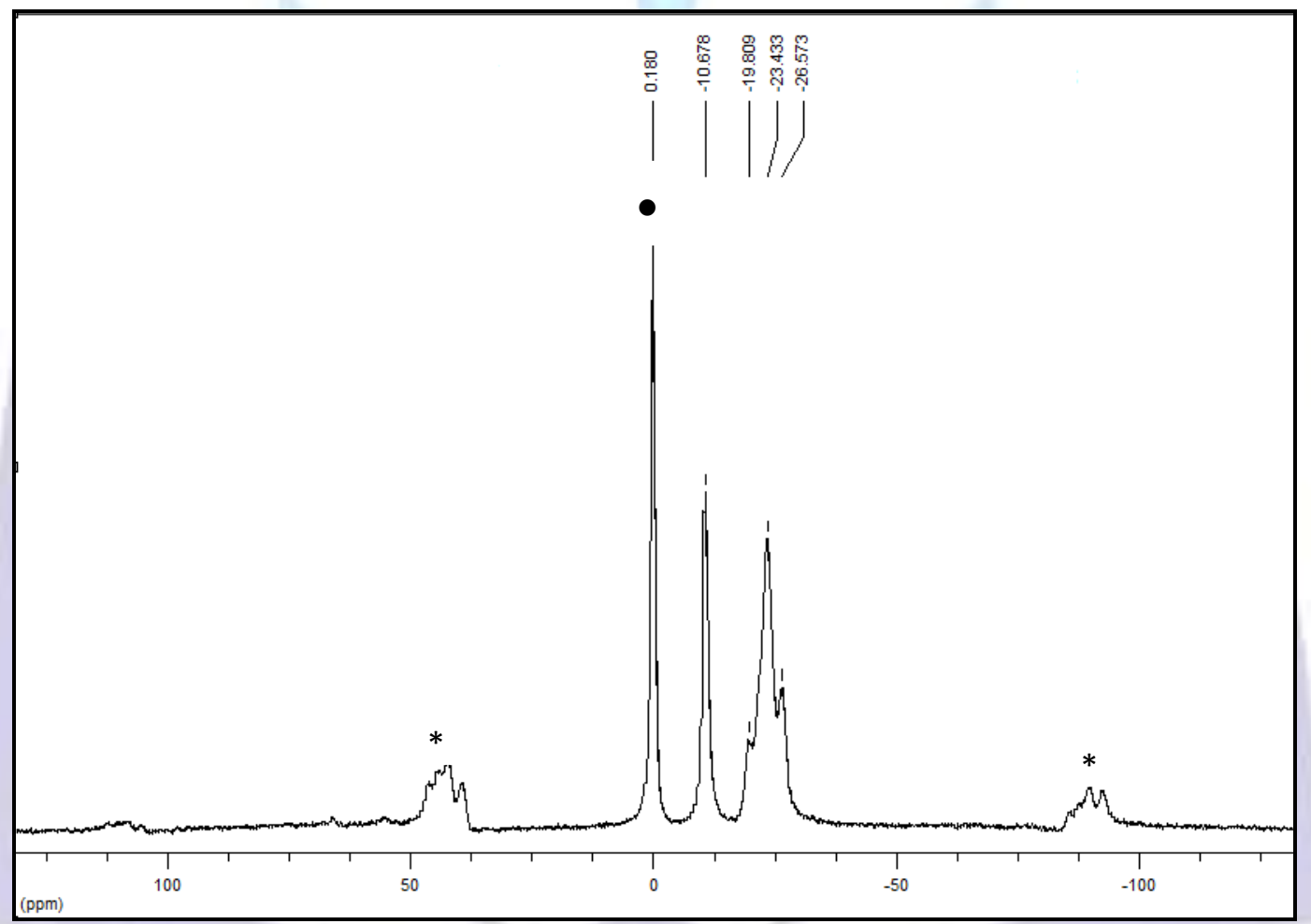

Figure 5: ${ }^{31} \mathrm{P}$ MAS-NMR spectrum of 2,3-( $\left.\left(\mathrm{CH}_{3}\right)_{2} \mathrm{C}_{6} \mathrm{H}_{3} \mathrm{NH}_{3}\right]_{6} \mathrm{P}_{6} \mathrm{O}_{18} \cdot 2 \mathrm{H}_{2} \mathrm{O} . \mathrm{H}_{3} \mathrm{PO}_{4}$ : Reference, * Spinning side bands.

\section{Thermal analysis}

Thermal analysis was performed, using the multimodule 92 Staram analyzer, from room temperature to $400^{\circ} \mathrm{C}$ at a speed of $5^{\circ} \mathrm{C} / \mathrm{min}$. The simultaneous TGA-DTA analysis curves (Fig.6) of $\left[2,3-\left(\mathrm{CH}_{3}\right)_{2} \mathrm{C}_{6} \mathrm{H}_{3} \mathrm{NH}_{3}\right]_{6} \mathrm{P}_{6} \mathrm{O}_{18} .2 \mathrm{H}_{2} \mathrm{O}$ exhibits a very intense endothermic peak, at $65^{\circ} \mathrm{C}$, on the DTA curve. Thermogravimetric analysis (TGA) shows that this peak is accompanied with a weight loss of $2.83 \%$ comparable to the theoretical loss $(2.9 \%)$ relative to the dehydration of the title material. the departure of water molecules at this low temperature is due to the thermal agitation of water molecules and their isolation in the network. The Series of DTA endothermic peaks occurring at $205^{\circ} \mathrm{C}, 224^{\circ} \mathrm{C}$ and $237^{\circ} \mathrm{C}$, accompanied by a significant weight loss, correspond to a pyrolysis of organic molecules. The resulting product is a consisting liquid of polyphosphoric acids contaminated of black carbon. This decomposition is occurred before the material melting. These phenomena are confirmed by DSC performed in the same temperature range (Fig 7). 


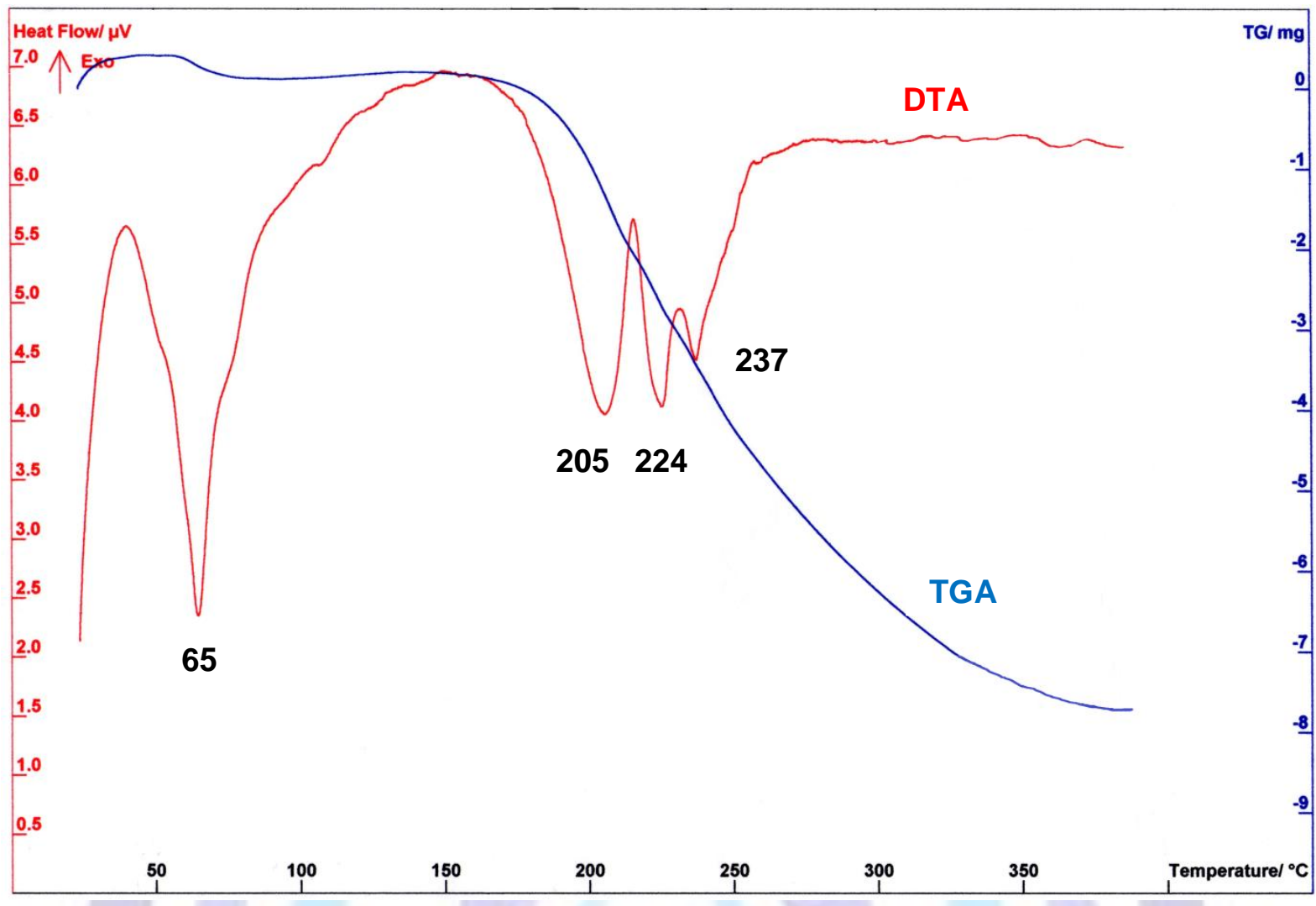

Figure 6: DTA and TGA curves of 2,3- $\left.\left(\mathrm{CH}_{3}\right)_{2} \mathrm{C}_{6} \mathrm{H}_{3} \mathrm{NH}_{3}\right]_{6} \mathrm{P}_{6} \mathrm{O}_{18} .2 \mathrm{H}_{2} \mathrm{O}$.

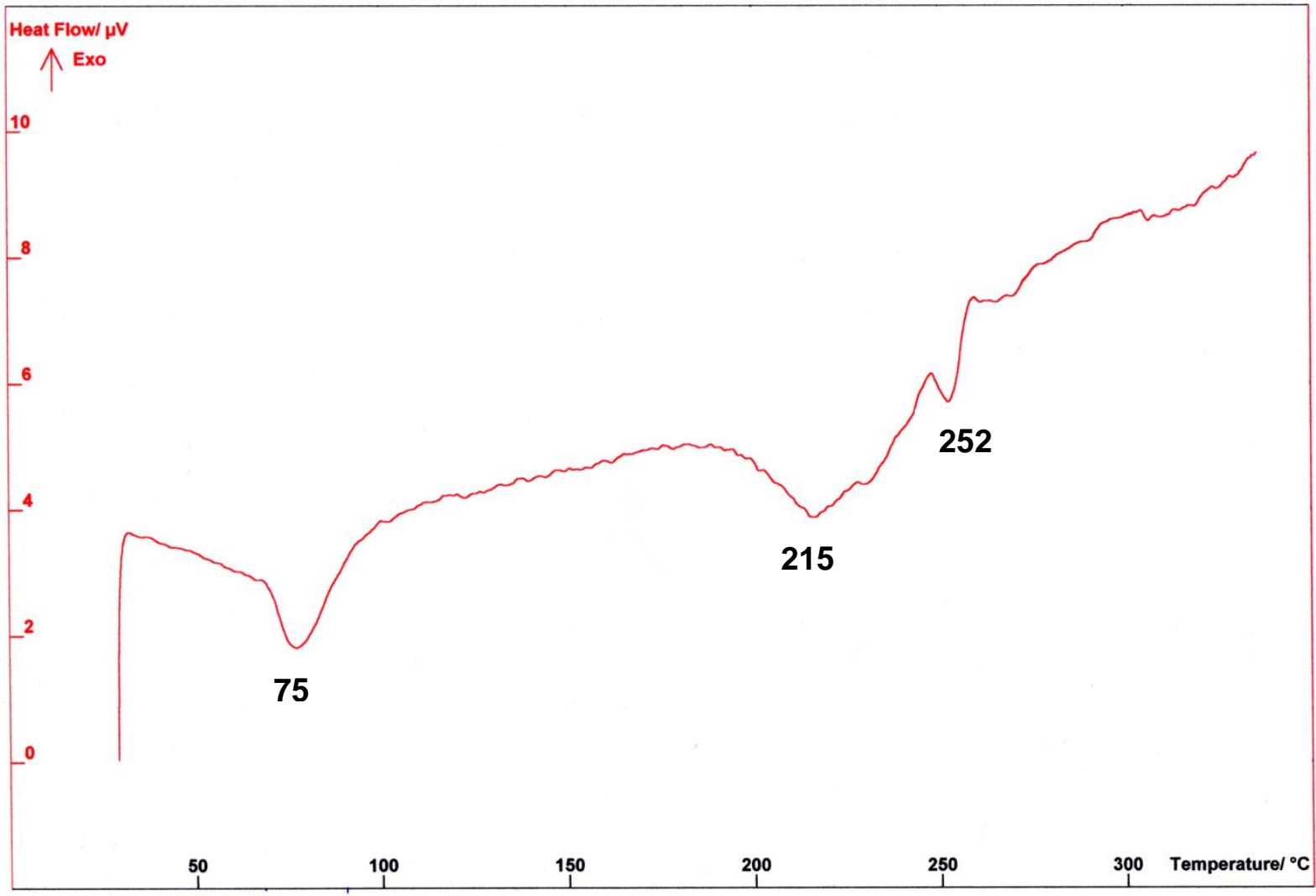

Figure 7: DSC curves of $\left[2,3-\left(\mathrm{CH}_{3}\right)_{2} \mathrm{C}_{6} \mathrm{H}_{3} \mathrm{NH}_{3}\right]_{6} \mathrm{P}_{6} \mathrm{O}_{18} \cdot 2 \mathrm{H}_{2} \mathrm{O}$. 


\section{IR Absorption Spectroscopy}

The infrared spectrum (Fig. 8) was recorded in the range 400-4000 $\mathrm{cm}^{-1}$ using the NICOLET IR 200 FT-IR infrared spectrometer. This spectrum exhibits characteristics bands of the different components of the analyzed product. The bands at 1247,1121 and $1069 \mathrm{~cm}^{-1}$ are characteristics of asymmetric and symmetric vibrations of OPO groups, those at $1032,952,780$ and $710 \mathrm{~cm}^{-1}$ are due to the asymmetric and symmetric vibrations of POP groups [8]. The intense bands at 1579 and $1600 \mathrm{~cm}^{-1}$ are characteristics of an aromatic ring, a band to $3449 \mathrm{~cm}^{-1}$ relating to the aromatic $\mathrm{NH}_{2}$ group. The bands at $780 \mathrm{~cm}^{-1}$ and $710 \mathrm{~cm}^{-1}$, corresponding to deformation vibrations of $\mathrm{C}-\mathrm{C}$ and $\mathrm{C}-\mathrm{H}$ groups, show that the aromatic ring is 1,2,3-trisubstituted. The large band at 2880 $\mathrm{cm}^{-1}$ and that at $1639 \mathrm{~cm}^{-1}$ correspond to the stretching vibration and deformation vibration respectively of water molecules. The assignment shows that the vibraionnal spectroscopic data are in agreement with the composition of single-crystal X-ray diffraction results.

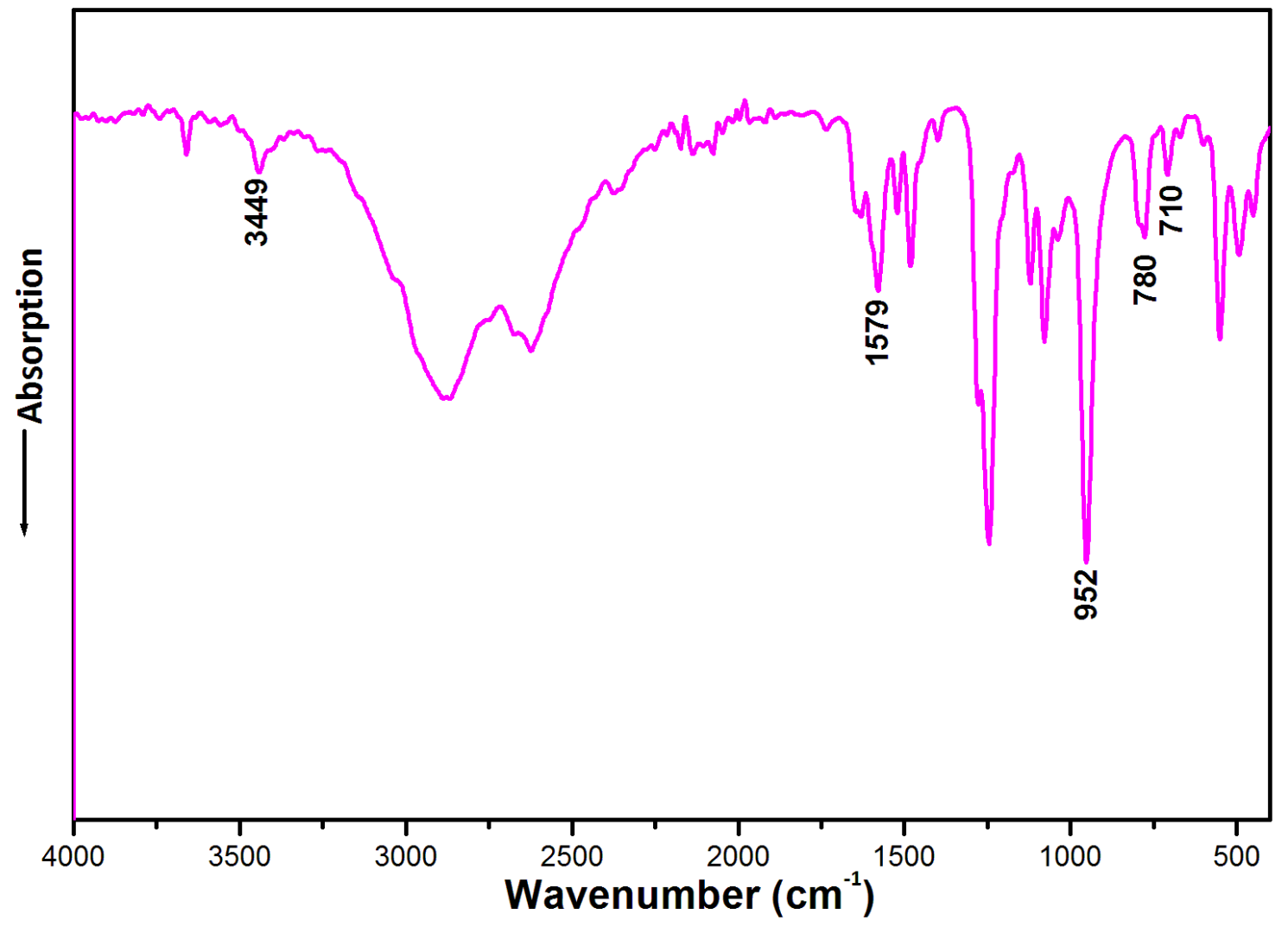

Figure 8: IR spectrum of $\left[2,3-\left(\mathrm{CH}_{3}\right)_{2} \mathrm{C}_{6} \mathrm{H}_{3} \mathrm{NH}_{3}\right]_{6} \mathrm{P}_{6} \mathrm{O}_{18} \cdot 2 \mathrm{H}_{2} \mathrm{O}$.

\section{REFERENCES}

[1] S. Mahesh, M. A. Green, and S. Natarajan, 2002, J. Solid State Chem. 165, 334.

[2] U. Schulke and R. Kayser, 1985, Z. Anorg. Allg. Chem., 531, 167.

[3] Walker, N. \& Stuart, D. (1983). Acta Cryst. A39, 158-166.J.

[4] J. M. Cense, 1990, Modelar Graphics for the Macintosh (Molview) in "Modelling of Molecular Structures and Properties" Elsevier, 763.

[5] L. J. Farrugia, Ortep 3 for Windows, 1997, J. Appl. Cryst. 30, 565.

[6] M. Bagieu-Beucher, M. T. Averbuch-Pouchot and M. Rzaigui 1991, Acta Cryst. C47, 1364.

[7] R. Bel Haj Salah, L. Khedhiri, C. Ben Nasr, M. Rzaigui and F. Lefebvre, 2010, Phosphorus, Sulfur, and Silicon.

[8] A. N. Lazarev and A. Mitgoroski. Izv. Akad. Nauk SSSR, Neorg. Mater, 1974, 10, 661. 